\title{
Performance Analysis of the Optimized Link State Routing Protocol with Link-Layer Feedback for Mobile Ad hoc Networks
}

\author{
Phu Hung Le \\ Hanoi University of Natural Resources and Environment, Vietnam
}

\begin{abstract}
In Mobile Ad hoc Network (MANET), routing is one of the important problems in research. Routing influence significantly the network performance. In this paper, we analyze performance of the Optimized Link State Routing Protocol with Link-layer Feedback (OLSR-FB) that is an improved protocol of OLSR protocol by comparing to the famous protocol DSR in terms of Packet Delivery Fraction, Delay, Routing overhead and Normalize Routing Load. Simulation results show that the OLSR-FB' packet delivery fraction outperforms that of the DSR in some cases. Our results also show that OLSR-FB“ Delay, Routing overhead and Normalize Routing Load are less than the corresponding results from the DSR.
\end{abstract}

Keywords: Mobile Ad hoc Networks; Routing Protocol; OLSR; DSR

\section{INTRODUCTION}

A Mobile Ad Hoc Network (MANET) is the network that wireless mobile nodes can freely and dynamically selforganize into arbitrary and temporary network topologies. An ad hoc network has not also any pre-existing communication infrastructure $[8,9,10,11]$.

MANET has many applications including disaster recovery situations, defence (army, navy, air force), healthcare, academic institutions, corporate conventions/meetings. In MANET, routing protocols are divided into three categories:

On-demand routing protocols are protocols that only calculate a path when they need data transmission. Some ondemand protocols are Ad hoc On-Demand Distance Vector (AODV) [3], Dynamic Source Routing (DSR) [4], Temporally Ordered Routing Algorithm (TORA) [5].

Contrary to On-demand routing protocols, in proactive (table-driven) protocols each node maintains the routing table and topology of network. These protocols have low delay when an application needs to send packets because a path to the destination is immediately available. Some famous proactive protocols are Optimized Link State Routing (OLSR) [1], Destination-Sequenced Distance-Vector (DSDV) [2].

And the third category is hybrid protocols that use both periodic and on-demand routing, for example, the Zone Routing Protocol (ZRP) [7] .

In this paper, we evaluate OLSR-FB[6] by comparing to the prominent protocol DSR. This paper is organized as follows. Section II and III introduce the detail structure of OLSR-FB and DSR. In section IV, we compare the OLSR-FB to the DSR and conclusion in section V.

\section{THE OPTIMIZED LINK STATE ROUTING PROTOCOL WITH LINK-LAYER FEEDBACK}

A. Topology information: In the OLSR protocol[1], a HELLO message in OLSR contains information about a node's neighbors and the current link status of a node. Nodes periodically broadcast HELLO message to detect their neighbors. OLSR uses control messages called Topology Control (TC) messages. Each node periodically sends a TC message in the network to declare its MPR Selector set. Nodes build the topology table based on information from TC message.

B. Multipoint Relay selection: Multipoint Relay (MPR) set consists of a subset of 1-hop neighbors which covers all the 2-hop neighbors. The MPR set needs be small enough to obtain the efficiency for multipoint relay. The MPR set is the red nodes in the following figure. 
Vol. 7, Issue 9, September 2018

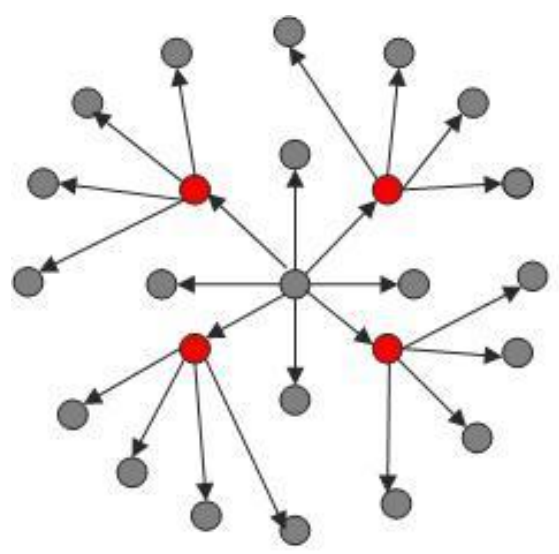

Figure 1: Multipoint Relay

C. Routing table calculation: Each node calculates and maintains a routing table that allows it to transmit the packets to other destinations in the network. The routing table is calculated based on the information in the neighbor table and the topology table. If any these tables are changed, the routing table is re-computed to update the route information. When a node detects a change in its neighborhood or a route is expired, the routing table is recalculated. The shortest-path algorithm is used to find the path from the source to the destination.

D. Link-layer Feedback: The protocol uses link-layer feedback. The link-layer will send a feedback to routing layer to inform failed links.

\section{THE DYNAMIC SOURCE ROUTING PROTOCOL}

The Dynamic Source Routing Protocol (DSR) is a well-known reactive protocol for mobile ad hoc network developed by David B. Johnson et al [4]. The DSR protocol uses two mechanisms that work together to discover and maintain source routes in the ad hoc network.

\section{A. Route Discovery}

In DSR, when node $S$ creates a new packet destined to other node D, it puts a source route giving the sequence of hops into the header of the packet. The packet can come to D based on this sequence of hops. Normally, $\mathrm{S}$ will take a suitable source route in its Route Cache of routes previously learned, but if Route Cache has no route, it will initiate the Route Discovery protocol to dynamically find a new route to D. In this case, S is called as the initiator and D as the target of the Route Discovery.

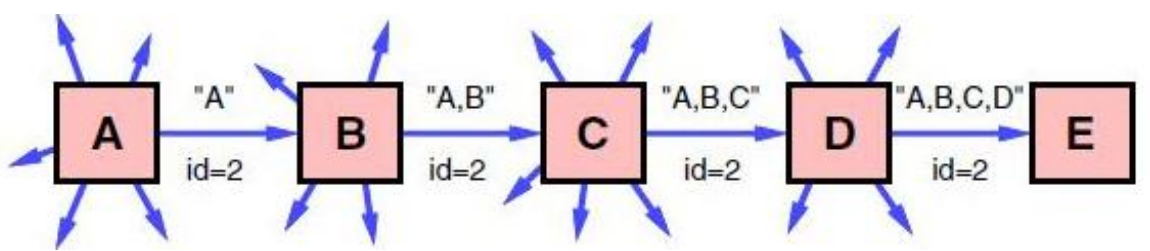

Figure 2: Route discovery

When initiating a Route Discovery, the sending node puts a copy of the original packet into a local buffer called the Send Buffer. While the Send Buffer has not had a source route to the packet's destination yet, it keeps a copy of each packet that cannot be transmitted by this node. The time that each packet was placed into the Buffer is stamped and is removed after residing in the Send Buffer for some timeout periods.

The FIFO or other replacement strategy can also be used to evict packets before they expire if it needs to prevent the Send Buffer from overflowing. While a packet is still in the Send Buffer, the node occasionally needs to initiate a new Route Discovery for the packet's destination address. However, the number of initiations of such new Route Discoveries for the same address must be limited since the destination node cannot be currently reachable. Specially, because of the limited wireless transmission range and the movement of the nodes in the network, the network may at times become partitioned. It means that there is currently no sequence of nodes through which a packet could be forwarded to reach the destination. Such network partitions may be rare or may be common because of depending on the movement pattern and the density of nodes in the network. To diminish the overhead from such Route Discoveries, the exponential back-off is used to limit the rate at which new Route Discoveries may be initiated by any node for the same target. 


\section{B. Route Maintenance:}

When a source route is used to originate or forward a packet, each node transmitting the packet is in charge of confirming that the packet has been received by the next hop along the source route. The packet is retransmitted (up to a maximum number of attempts) until this confirmation of receipt is received. For example, in the situation depicted in Figure 3.4, node A has created a packet for E using a source route via intermediate nodes B, C, and D. In this case, node $\mathrm{A}$ is in charge of receipt of the packet at $\mathrm{B}$, node $\mathrm{B}$ is in charge of receipt at $\mathrm{C}$, node $\mathrm{C}$ in charge of receipt at $\mathrm{D}$, and node $\mathrm{D}$ is in charge of receipt finally at the destination $\mathrm{E}$. If no receipt confirmation is received when the packet is retransmitted by some hop the maximum number of times, this node sends a ROUTE ERROR message to the original sender of the packet, specifying the link over which the packet could not be forwarded. For example, in Figure 3.4, if $\mathrm{C}$ is impossible to deliver the packet to the next hop $\mathrm{D}$, then $\mathrm{C}$ returns a ROUTE ERROR to A, announcing that the link from C to D is currently "broken". Node A then deletes this broken link from its cache. Any retransmission of the original packet is a function for upper layer protocols such as TCP.

To send such a retransmission or other packets to this same destination E, if A get in its Route Cache another route to E (for example, from additional ROUTE REPLYs from its earlier Route Discovery, or from having overheard sufficient routing information from other packets), it can immediately send the packet using the new route. Otherwise, it may address a new Route Discovery for this target.

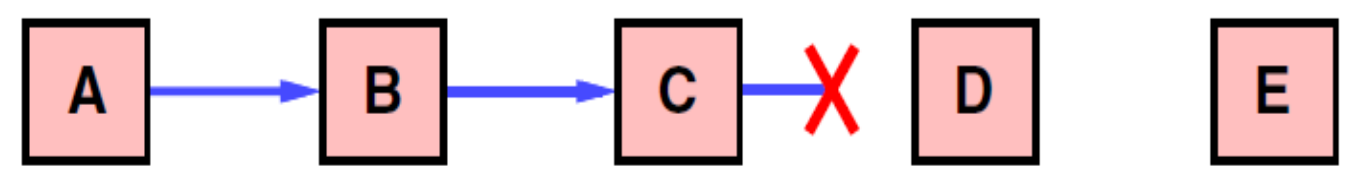

Figure 3: Route maintenance

\section{PERFORMANCE EVALUATION}

\section{A. Simulation environment}

We implement protocol in NS-2 with $11 \mathrm{Mbps} 802.11$ channels. The traffic source is Constant Bit Rate (CBR). The distributed coordination function (DCF) of IEEE 802.11 for wireless LANs is used as the MAC layer. The Random Waypoint and Two-Ray Ground models have been used as propagation model and mobility model, respectively. 40 nodes is used and they move within an area of $550 \mathrm{~m} \times 550 \mathrm{~m}$.

\section{B. Simulation results}

In the simulations, we compare the performance between OLSR-FB and DSR for:

1-Packet delivery fraction (PDF)

2-Delay

3- Routing overhead

4-Nomalize Routing Load (NRL)

As shown in Figure 4, the PDF of DSR outperforms that of OLSR-FB with 8 and 10 connections. However, at 13 and 15 connections, the PDF of OLSR-FB is higher than the DSR.

$$
\text { 口OLSR_FB GDSR }
$$

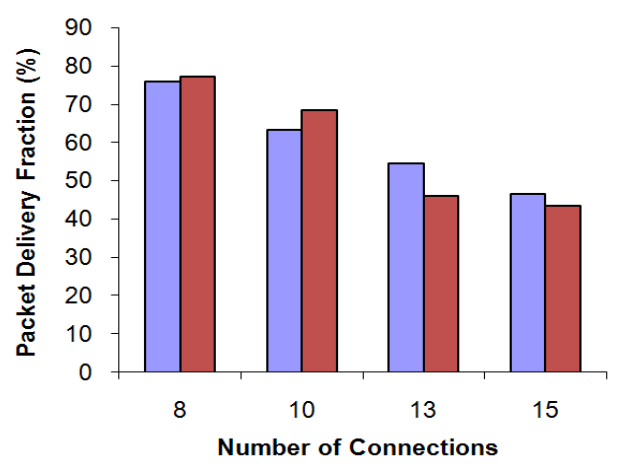

Figure 4: Packet delivery fraction 
In Figure 5, the delay of OLSR-FB reduces significantly compared to that of DSR. When the number of connections increases the delay of DSR rises fast.

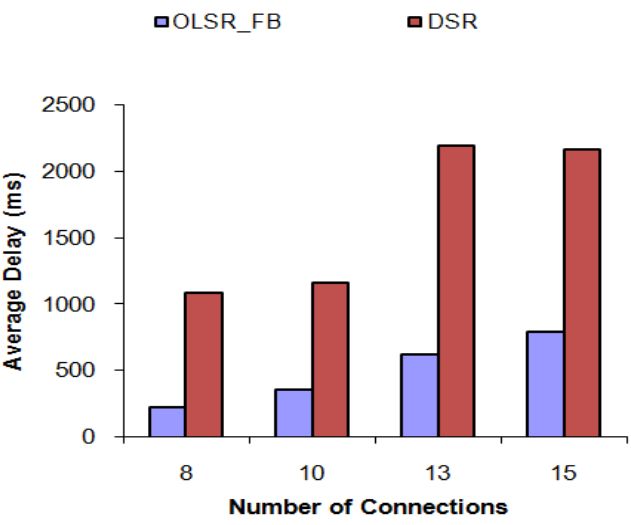

Figure 5: Delay

We can see in Figure 6 that Routing overhead of two protocols increases when the number of connections increases. However, Routing overhead of OLSR-FB is less than that of DSR.

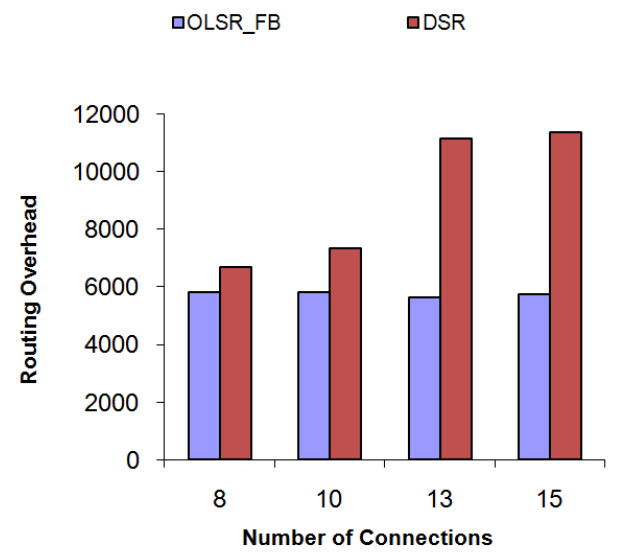

Figure 6: Routing overhead

When the number of connections rises Nomalize Routing Load of DSR increases significantly. Nomalize Routing Load of OLSR-FB is much less than that of the DSR as shown in Figure 7.

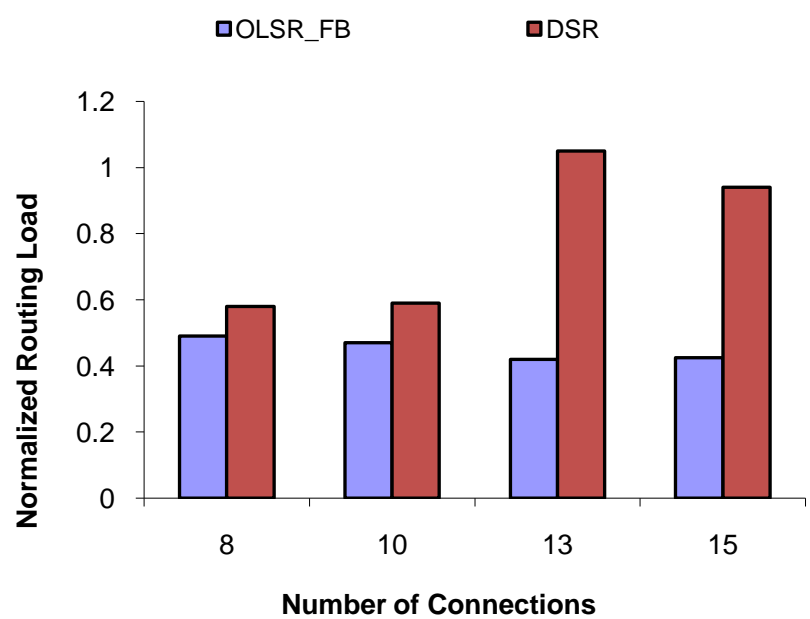

Figure 7: Nomalize Routing Load 


\section{IJARCCE}

\section{CONCLUSION}

In this paper, we compare the Optimized Link State Routing Protocol with link-layer feedback(OLSR-FB) that is a improved protocol of OLSR to DSR. We can see that in term of the Packet delivery fraction, OLSR-FB is better than DSR when the number of the connections increases and on the contrary DSR is better. In terms of Delay and Routing overhead and Nomalize Routing Load, OLSR-FB is lower than DSR.

\section{REFERENCES}

[1]. T. Clausen, P. Jacquet, IETF Request for Comments: 3626, Optimized Link State Routing Protocol OLSR, October 2003

[2]. Perkins CE, Bhagwat P. Highly dynamic destination-sequenced distance-vector routing (DSDV) for mobile computers. In Proceedings of ACM Sigcomm, 1994.

[3]. C. E. Perkins and E. M. Royer. Ad Hoc On Demand Distance Vector (AODV) Routing. draft-ietf-MANET-aodv-02.txt, Nov. 1998. (work in progress).

[4]. David B. Johnson, David A. Maltz, Josh Broch, "DSR: The Dynamic Source Routing Protocol for Multi-Hop Wireless Ad Hoc Networks," Ad Hoc Networking, Addison-Wesley, 2001:139 172.

[5]. Park and S. Corson, "Temporally-ordered routing algorithm (TORA) version 1 functional specification", IETF Draft: draft-ietf-MANET-toraspec-04.txt, 2001.

[6]. https://cloudns2.wordpress.com/um-olsr-patch/

[7]. Haas and Pearlman Zone Routing Protocol,1997.

[8]. Phu Hung Le, Guy Pujolle, Thi-Mai-Trang Nguyen, "An Interference-aware multi-path routing protocol for Mobile Ad hoc Network", proc of ICNSC 2011, Delft, the Netherlands, April 2011, IEEEXplore, pages 503 - 507.

[9]. Phu Hung Le, Guy Pujolle, "A Link-disjoint interference-aware multi-path routing protocol for Mobile Ad hoc Network", proc of The International Conference on Digital Information and Communication Technology and its Applications(DICTAP 2011), Dijon, France, June 2011, Springer LNCS, pages 649-661.

[10]. Phu Hung Le, "A Performance Evaluation of Multi-Path Routing Protocols for Mobile Ad Hoc Network", the 10th IEEE/IFIP International Conference on Embedded and Ubiquitous Computing, Paphos, Cyprus, December 2012

[11]. Phu Hung Le "A New Efficient Multi-path Routing Protocol for Mobile Ad hoc Networks", International Journal of Advanced Research in Computer and Communication Engineering, 2017.

\section{BIOGRAPHY}

Phu Hung Le received the Ph.D degree in Computer Science from Pierre and Marie Curie University (Paris 6 University), France. Phu Hung Le is the author of many articles and is a reviewer for many international conferences and journals. His research interests are Mobile Ad hoc Networks and Sensor Networks. 